\title{
NUMERICAL SIMULATIONS OF PLANETARY SYSTEMS OF THE
}

\section{JUPITER-SATURN TYPE}

\author{
R.A.BROUCKE \\ Department of Aerospace Engineering and Engineering Mechanics, \\ University of Texas at Austin, USA
}

\begin{abstract}
We made a numerical study of the General Three-Body Problem in two dimensions, with the intention to obtain some statistical estimates of the outcome of the system after a long time. Two different sets of masses were used. In the first series of experiments we use masses in the ratio of $0.95,0.04$ and 0.01 . In the second series, we use masses that are exactly in the Sun-JupiterSaturn ratio. To facilitate the discussion, we use the names Sun, Jupiter and Saturn for the three masses, in both cases. In all our experiments, the orbit of Jupiter starts with zero eccentricity and with a unit radius. However, the orbit of Saturn varies in two ways: the initial value of the semimajor axis varies from 1.1 to 3.5 and the eccentricity from 0.0 to 0.75 . In total about 4000 cases were run for the two series of masses. All the numerical integrations were done with the method of recurrent power series of order 14, in a heliocentric frame of reference, integrating thus eight simultaneous first-order differential equations. All integrations were performed for a maximum of 12,500 canonical units of time, corresponding to about 2000 revolutions of Jupiter. The cause of termination or type of catastrophe for the system has been determined in all cases. In most cases, this is a close approach of Saturn with Jupiter, followed by ejection of Saturn from the system.
\end{abstract}

\section{Numerical investigation of a typical planetary system} $(0.05,0.04,0.01)$

We first investigated the configuration with masses $(0.95,0.04,0.01)$. In other words, the sun would have a mass $m=0.95$, the planets $m_{1}, m_{2}$ have the masses 0.04 and 0.01 , about in the Jupiter-Saturn ratio. We also assume that $m_{1}$ is the inner planet (the largest mass!) and $m_{2}$ the outer planet. Our units of length are normalized in such a way that the initial value of the semi-major axis $a_{1}$ of the inner planet are always equal to 1 .

As an initial rough estimate of the stability of this system (on the basis of the opening of the Hill zero-velocity curves), we computed the $C^{2} E$-value of several orbits to determine for which cases the relative equipotential lines would be closed or open, considering that in the case where these curves are open at the inner libration point $L 1$, there is possible exchange or collision between the bodies. The critical value of $C^{2} E$ is here $-0.58820017 \mathrm{E}-4$. All orbits with a value of $C^{2} E$ larger than the critical value may have exchange. Note that the above number depends solely on the three masses. We find that the stability boundaries for Saturn are $a=1.18$ and $a=2.58$. When the semi-major axis $a$ is less than 1.18 , we have a satellite of Jupiter that can not escape. When $a$ is larger than 2.58 , we have a planet that can not have exchange with Jupiter. Here $E$ represents the energy and $C$ the angular momentum of the system (see Szebehely and Zare, 1977).

To obtain more insight regarding the types of planetary systems that can be stable and survive for long periods of time, we planned a series of computer experiments to give us a collection of data from which we might derive some initial statistical information and preliminary conclusions.

The most important feature of the present set of computer runs is that we keep track, in a very systematic way, of the mode of termination of each run. In 


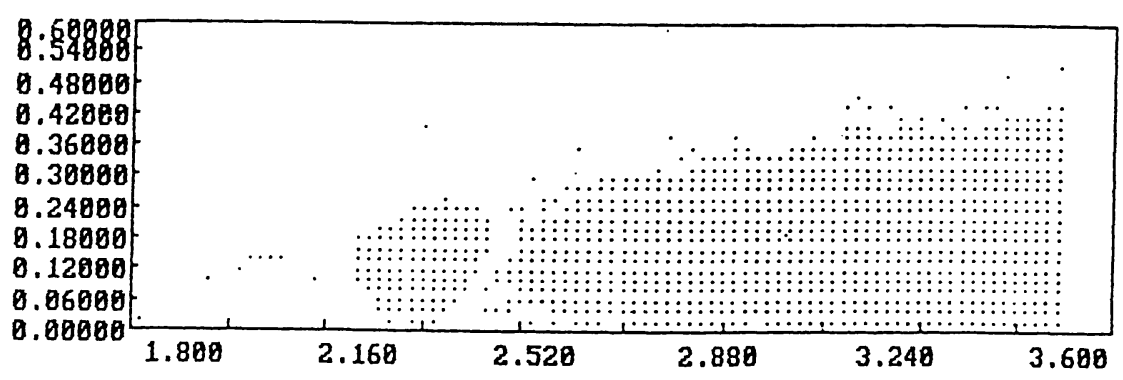

Fig. 1.

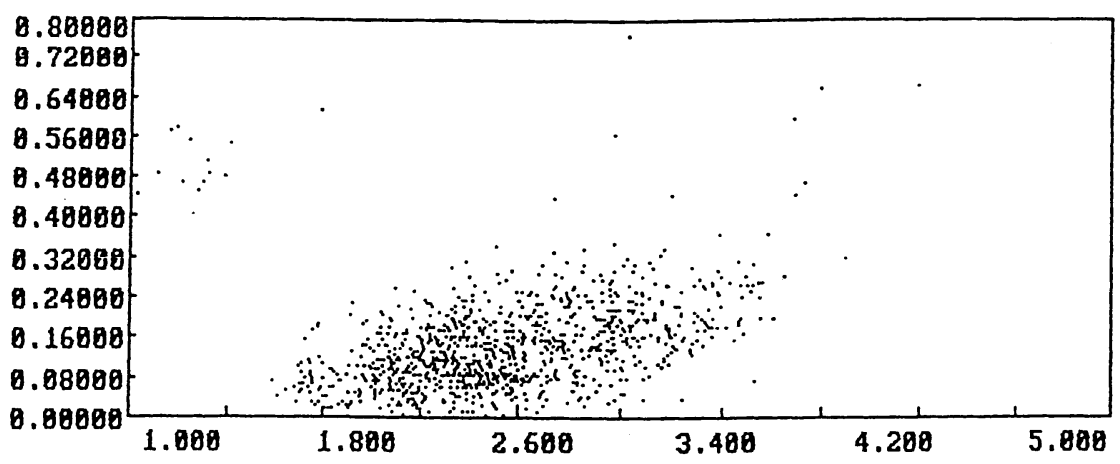

Fig. 2.

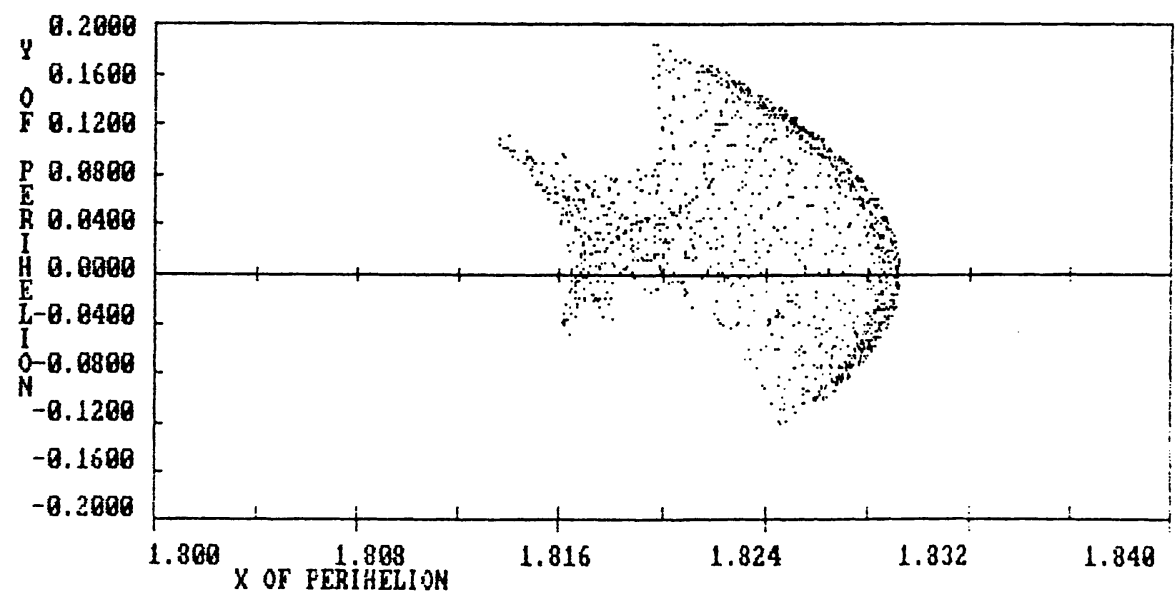

Fig. 3. 
other words, a set of codes (integer numbers) is defined for the different possible determinations. This code and the end condition for each simulation are saved on a summary file for later analysis.

The experiment consists in the integration of a large number of so-called SunJupiter-Saturn systems, with a fixed set of masses. The integrations are performed in the heliocentric frame, with the sun at the origin $(0,0)$. We limited ourselves to the two-dimensional (planar) case. The initial conditions for Jupiter are fixed $\left(a_{j}=1.0, e_{j}=0.05\right)$ while the initial values for Saturn have been varied: $a_{8}$ from1.1 to 3.5 and $e$, from 0 to 0.62 (where $a=$ semi-major axis and $e=$ eccentricity).

There is thus a total of 3872 cases. Each of these cases was run with a maximum final time of 12,500 canonical units (about 2000 revolutions of Jupiter) and a maximum of 100,000 steps. Eighteen end-conditions are tested after every integration step. Figure 1 shows the set of initial conditions $a_{s}, e_{8}$ that terminates with the codes 2 or 3 . In other words these orbits reach the final allowed integration time or number of the integration steps. Therefore we will call these orbits stable, although this conclusion should be considered as preliminary at this point. Most of the orbits survive the total integration time. These orbits are the most stable. A few orbits had reached 100,000 integration steps. They seem to have close approaches between two of the three bodies and are thus not so stable. These orbits will be analyzed further.

The next important question is, of course: how about all the other orbits? An analysis of the termination codes shows that in almost all cases we have a close approach between Jupiter and Saturn, usually followed by an ejection of Saturn from the system.

Figure 1 seems to indicate a gap in the group of stable orbits, $a_{s}=2.4$ (and for all eccentricities). This gap divides thus the collection of stable orbits in two groups. The largest group has $a_{3}>2.4$ while there is a smaller stable group with $2.2<a_{s}<2.4$. There also are a few isolated stable orbits near $a_{s}=2.0$ and $e_{s}=0.15$, which will be studied in more detail.

An even more interesting diagram is given in figure 2 . It shows the final value of the Saturn semi-major axis and eccentricity. This collection of points could be considered a sample of nearly random initial values that lead to stable orbits. We also see a few isolated stable orbits with high eccentricities: near $a_{s}=1.3$ and $e_{s}=0.50$. We are in process of studying these results in more detail.

\section{The location of the perihelion of Saturn}

A series of experiments was made to determine the motion of the perihelion of the smallest planet (Saturn). It is very important to know if the perihelion "librates" or "circulates". It is very important to know the region of space where the perihelion is located because this is intimately related to the existence of resonances. Therefore we made a few computer runs for the sole purpose of plotting the location of the perihelion of the smallest planet in physical $x y$-space. The perihelion distance being $a(1-e)$, the coordinates of the instantaneous (osculating) perihelion locations are $x_{p}=a(1-e) \cos \omega ; y_{p}=a(1-e) \sin \omega$.

The plots are remarkably instructive because they visualize the interaction of 
three important orbit elements simultaneously $(a, e, \omega)$. In figure 3 , we show an example of a plot giving the location of the perihelion of Saturn, plotted with isolated dots, at the rate of one dot per integration step. We clearly see that the perihelion is confined to and librates in a well-defined small region of the plane.

\section{The Sun-Jupiter-Saturn system with masses $(0.998761166,0.00095360,0.000285230)$}

The critical $C^{2} E$-value is $-0.95543 \mathrm{E}-9$, for the masses of Jupiter and Saturn. For the initial values of the real semi-major axis and eccentricity that we used, the actual value of the $C^{2} E$-parameter is $-0.98988 \mathrm{E}-9$, which is slightly lower (by about $4 \%$ ). The conclusion of these numbers is that Saturn must always remain a planet of the system: it cannot become a satellite of Jupiter (and form a close binary with Jupiter). However, escape of the planet Saturn from the system is still not excluded! The stability boundaries for Saturn are $a_{2}=1.007$ and $a_{2}=1.31$. In other words, if the Saturn semi-major axis value were within these two boundaries, we would have possible exchange of type of motion: the bottleneck of the Hill curves is open and Saturn could become a satellite of Jupiter or remain a planet in orbit around the sun. An object at a distance $a_{2}$ from 1.0 to 1.007 is a satellite of Jupiter and cannot escape. An object with a value of $a_{2}$ greater than 1.31 can only be in a planetary orbit and can never be captured by Jupiter. This is the case for the real Saturn which is at $a_{2}=1.835$. We will report later on the detailed numerical explorations.

\section{Acknowledgements}

We want to thank Dr. David Black, director of the Lunar and Planetary Institute in Houston, for suggesting this study to us, as a continuation of his similar research (Graziani and Black 1981; Black, 1982).

\section{References}

Black, D.C.: 1982 Astron. J. 87, 1333-1337.

Graziani, F. and Black, D.C.: 1981 Astrophys. J. 251, 337-341.

Szebehely, V. and Zare, K.: 1977 Astron. and Astrophys. 58, 145-152.

\section{Discussion}

Cl.Froeschlé - Concerning computations of LCEs do you use another method than the Benettin-Galgani-Strelcyn, which appeared to us very efficient.

R.A.Broucke - I basically follow the standard method which consists in integrating the linearized equations of motion and performing a Gram-Schmidt re-orthogonaliza-tion at regular time intervals. However, I use a matrix which is of smaller dimension than in classical approach. I eliminate the time and I use the energy equation to reduce the order of the system of variational equations. For instance, in the Lorenz system or in the restricted problem of three bodies, I work with a $(2 \times 2)$-matrix, which gives me the two non-trivial Lyapunov exponents. The zero Lyapunov exponent is not computed. 